\title{
Ectopic thyroid as multiple nodules in bilateral lung lobes: a case report
}

\author{
Ling Cheng, Xudong Jiang, Yueqiang Jiang \\ Department of Geriatrics, Tongji Hospital, Tongji Medical College, Huazhong University of Science and Technology, Wuhan 430030, China \\ Correspondence to: Yueqiang Jiang. Department of Geriatrics, Tongji Hospital, Tongji Medical College, Huazhong University of Science and \\ Technology, Jiefang Road 1095\#, Wuhan 430030, China. Email: jyqwh@163.com.
}

\begin{abstract}
Ectopic thyroid rarely occurs in the thoracic or abdominal cavity, especially in the lung. Here, we describe an intrapulmonary thyroid tissue case in bilateral lung lobes without thyroid malignant history, which was found during a routine physical examination. A 37-year-old Chinese female showed multiple pulmonary nodules appearing in bilateral lung lobes on a computed tomography (CT) scan during a routine medical examination. To rule out the possibility of lung metastasis from occult cancer, a percutaneous lung biopsy was performed on a larger nodule. Histopathological examination confirmed that the nodule was completely consistent with normal thyroid tissue, which prevented the patient from requiring any unnecessary treatment. During the patient's follow-up, there was no abnormal thyroid function, and there was no change in the size of the nodule in the lung during serial CT scan. Currently, there is no guidance or consensus on the diagnosis of ectopic thyroid with bilateral multiple pulmonary nodules due to its rarity in clinical practice. When such pulmonary nodules are encountered, very careful diagnosis and follow-up should be performed.
\end{abstract}

Keywords! Ectopic thyroid; pulmonary nodule; case report

Submitted Dec 12, 2019. Accepted for publication May 11, 2020.

doi: 10.21037/gs-19-499

View this article at: http://dx.doi.org/10.21037/gs-19-499

\section{Introduction}

The thyroid gland is the first endocrine gland to develop during embryogenesis in third or fourth week of pregnancy. During development, the thyroid migrates from the endoderm protrusion of the first and second pharyngeal arch and descends anteriorly along the midline from the foramen caecum. However, ectopic thyroid, known as abnormal migration of the thyroid gland during development, is an uncommon thyroid congenital abnormality (1). According to reports, the prevalence of ectopic thyroid gland is 1 in every 100,000 to 300,000 in the general population, and the incidence is much higher in women than in men (2). Usually, the ectopic thyroid tissue occurs along the midline and rarely in the periphery. Rarely, ectopic thyroid may also occur in the thoracic, abdominal or pelvic cavity, such as in the lung, gall bladder, adrenal gland, or liver, which may be associated with abnormal migration or ectopic differentiation of the undefined endoderm cells (3-7). Most cases are asymptomatic and discovered during routine physical screening, when treating other diseases or at autopsy. However, symptoms may also occur, such as when the tumour is excessively large and related to surrounding tissues or organs. At this time, surgery or radioactive iodine ablation may be considered; however, it must be considered that the ectopic thyroid may be the only effectively functioning thyroid tissue (8).

To the best of our knowledge, the occurrence of an ectopic thyroid gland in the lung is extremely rare in the clinic, with few cases reported in the literature; ectopic nodules in the lung are almost always in a single lobe or homolateral lobes and rarely occur in lobes on both sides of the lung $(3,9)$. Meanwhile, most patients with ectopic thyroid in the lung usually present as euthyroid (2). Herein, we describe a case of ectopic thyroid that occurred in bilateral lung lobes with normal function of thyroid 

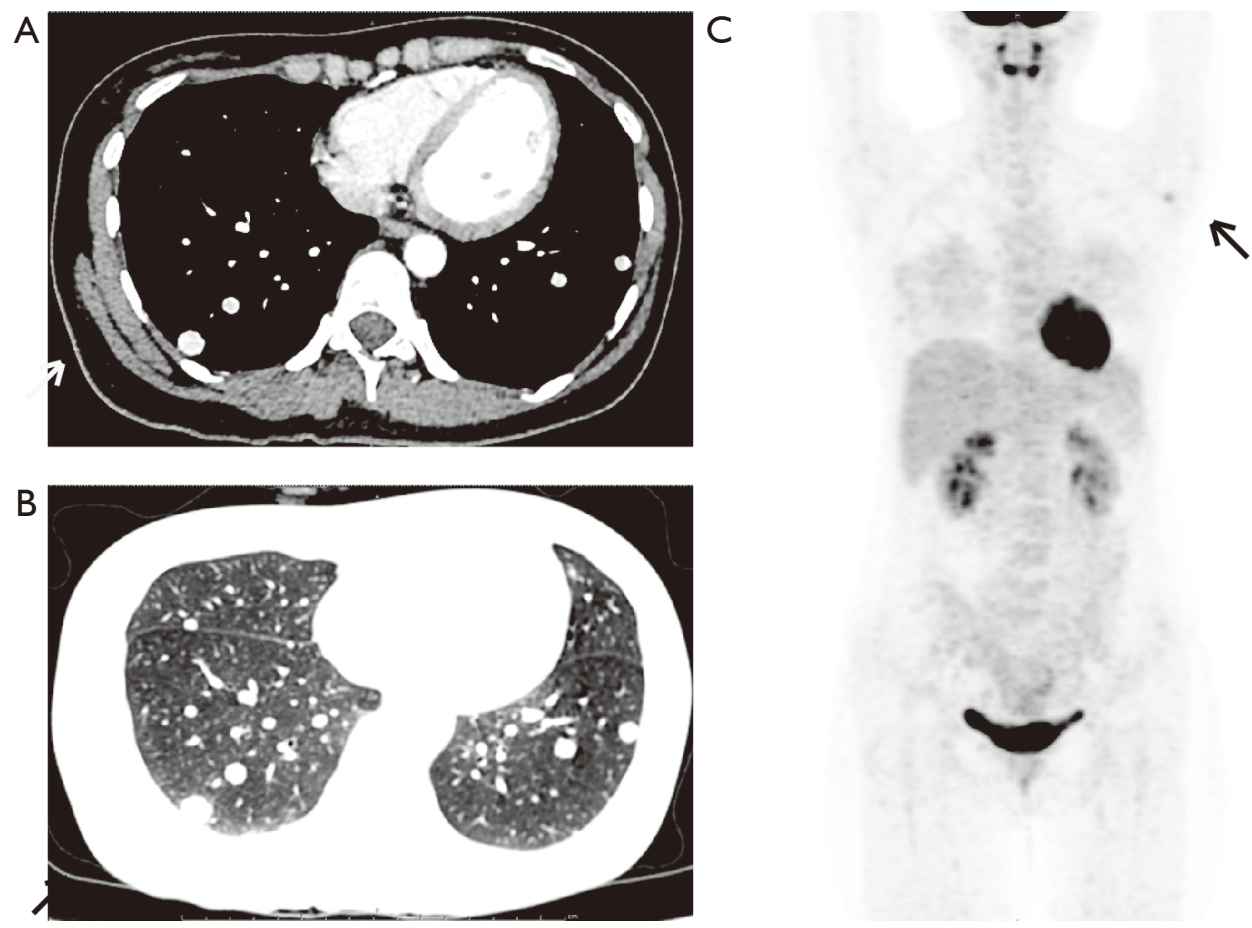

Figure 1 The results of pulmonary computed tomography (CT) scan and positron emission tomography-computed tomography (PETCT) examination show abnormalities in the lungs. On the lung window image (A) and the longitudinal window image (B) of the pulmonary CT scan, some well-defined, round nodules are seen in the bilateral pulmonary lobes, and one 15-mm-sized nodule is revealed beneath the pleura (arrow). (C) PET-CT showed the most obvious site of increased metabolism in the axillary lymph nodes (black arrow), with multiple nodules in bilateral lung lobes with mildly increased metabolism.

hormone and without any history of malignancy. After excluding the diagnosis of metastatic carcinoma, the patient remains under careful and routine follow-up and has been for over a year and is still in good condition. Here we present the following article in accordance with the CARE reporting checklist (available at http://dx.doi.org/10.21037/ gs-19-499). (10).

\section{Case presentation}

A 37-year-old Chinese woman came to Tongji Hospital on June 2019 with the main complaint of abnormally elevated carbohydrate antigen 125 (CA125) levels of $37.3 \mathrm{U} / \mathrm{mL}$ (normal range, $0-35 \mathrm{U} / \mathrm{mL}$ ) detected in a routine medical screening. The patient had no history of malignancy, tuberculosis, alcohol or tobacco consumption. Upon review of the family history of the patient, no special medical history was found. The patient had a normal delivery after a full-term pregnancy, with no history of gynaecological or obstetric issues. The patient was generally in good condition without symptoms of weakness, such as anaemia, dyspnoea, fever, or weight loss. Physical examination indicated an enlarged axillary lymph node on the left side, without pain. No mass could be appreciated in either breast. No thyroid mass or nodule was palpated. The patient's thyroid function tests indicated euthyroid, with serum thyroid stimulating hormone (TSH) $3.049 \mu \mathrm{IU} / \mathrm{mL}$ (normal range, $0.30-4.94 \mu \mathrm{IU} / \mathrm{mL}$ ), free triiodothyronine (FT3) $2.73 \mathrm{pg} / \mathrm{mL}$ (normal range, $1.71-3.71 \mathrm{pg} / \mathrm{mL}$ ) and free thyroxine (FT4) $0.78 \mathrm{ng} / \mathrm{dL}$ (normal range, $0.70-1.48 \mathrm{ng} / \mathrm{dL}$ ). Pulmonary computed tomography (CT) showed multiple nodules in bilateral lung lobes that were well-defined, round and various-sized, with a maximum diameter of approximately $15 \mathrm{~mm}$ (Figure 1A,B). Meanwhile, B-ultrasound indicated a low echoic area of $7 \mathrm{~mm} \times 4 \mathrm{~mm}$ in the left breast and no abnormality in the thyroid gland. Magnetic resonance imaging (MRI) did not suggest any significant abnormalities in the patient's abdominal and pelvic cavities except for a small amount of fluid in the Douglas cavity, which might explain the slightly increased serum CA125 level. To clarify the reason for the 
elevated CA125 and to exclude multiple metastasis to the lung by occult malignancy, the patient underwent a positron emission tomography-computed tomography (PET-CT) examination. The results of the PET-CT showed multiple

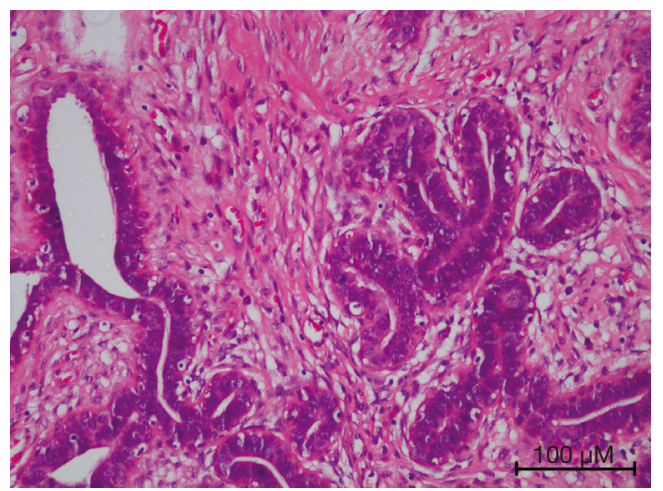

Figure 2 Microphotograph of the axillary increased lymph nodes. Hematoxylin and eosin (H\&E) show to be axillary fibroma adenoma (magnification $\times 200$ ). nodules in bilateral lung lobes with mildly increased metabolism (SUVmax 1.3) and a $1.3 \mathrm{~cm} \times 0.9 \mathrm{~cm}$ axillary lymph node with increased metabolism (SUVmax 2.4) on the left side (Figure 1C). No metabolic abnormalities were observed in other sites of the patient's body.

Combined with the patient's medical history, we first considered that the pulmonary nodules might be metastasis from breast cancer with enlarged metastatic axillary lymph nodes. Therefore, a left side axillary lymph node biopsy was performed. However, pathology of the lymph node was verified to be axillary fibroma adenoma (Figure 2). Then, a lung puncture biopsy was performed. Interestingly, pathology showed pulmonary alveolar tissue and thyroid follicular tissue without cellular atypia (Figure 3A). There was no evidence of malignancy in the microscopic findings. Immunohistochemical (IHC) staining indicated positive thyroglobulin (TG), which strongly suggested an origin of normal thyroid tissue (Figure 3B). Meanwhile, IHC staining of thyroid transcription factor-1 (TTF-1) revealed a positive reaction in the nucleus of the follicular cells
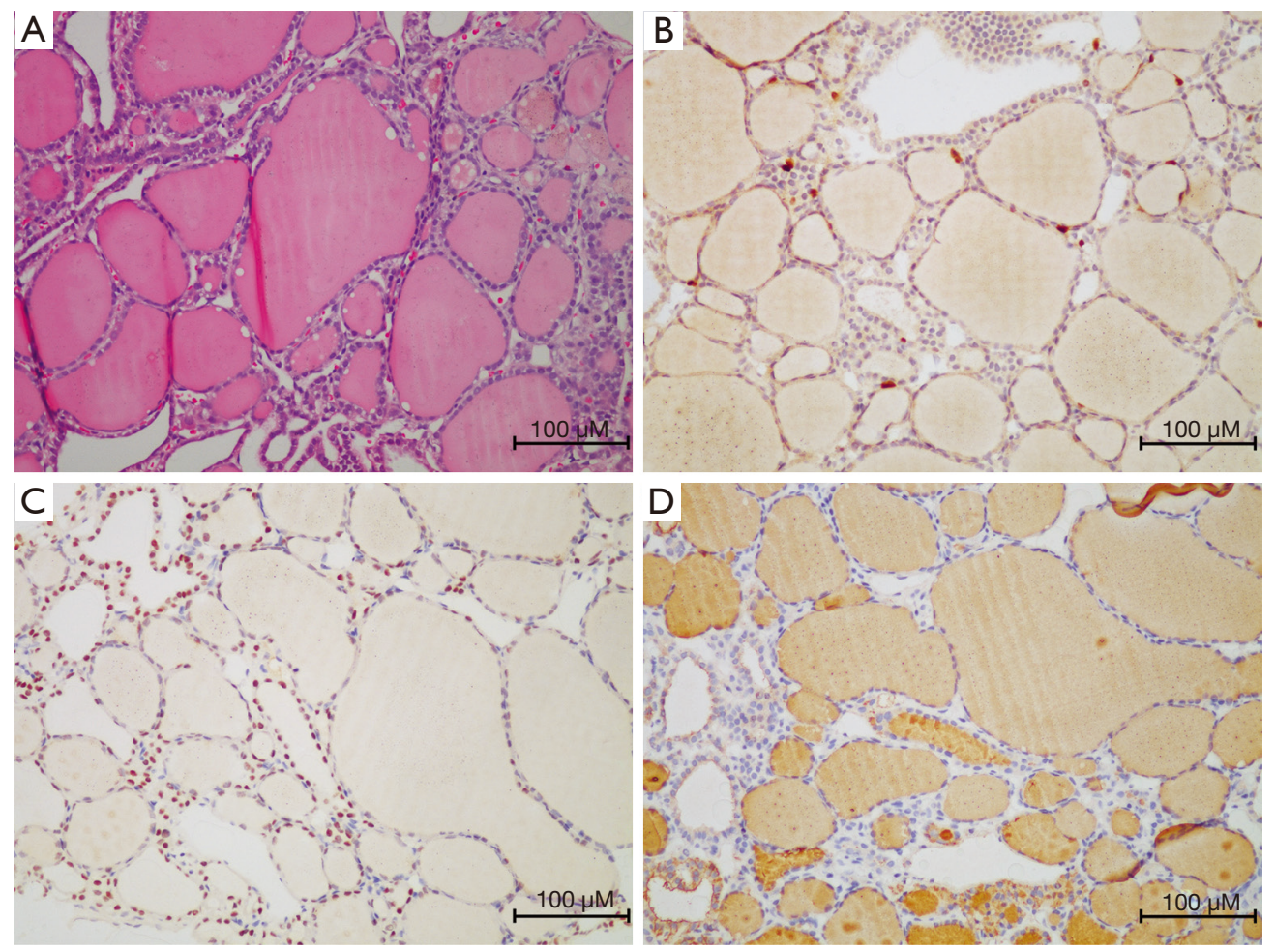

Figure 3 Microphotograph of thyroid ectopia in the nodules in her bilateral lung lobes. (A) H\&E show typical thyroid tissue with follicles containing colloid in the pulmonary parenchyma (magnification $\times 200$ ). (B) Positive immunohistochemistry staining for thyroglobulin (TG, magnification $\times 200$ ). (C) Positive immunohistochemistry staining for thyroid transcription factor-1 (TTF-1, magnification $\times 200)$. (D) The immunohistochemistry staining of follicular epithelial cells showed not strong positive for Ki67 (magnification ×200). 
(Figure 3C). IHC staining of Ki67, an index related to tumour proliferation capacity, showed no significant positivity (Figure 3D). Unexpectedly, during the percutaneous lung biopsy procedure, the patient suddenly developed uncontrolled haemoptysis and recovered after emergent treatment. Based on the lack of evidence of malignancy and after discussing with the patient and her family, a strategy of "watch and wait" was chosen for the patient. As of today, the patient has been followed up for over 1 year. During the follow-up period, the patient's serum CA125 level ranged from 37-59 U/mL, which was no more than twice the normal upper level value (35 $\mathrm{U} / \mathrm{mL}$ ), and pulmonary CT showed no changes in the pulmonary nodules in the bilateral lobes. Meanwhile, the patient's thyroid hormone levels remained in normal ranges.

\section{Discussion}

Our manuscript introduced a novel ectopic thyroid that occurred in bilateral lung lobes by accidental physical screening for other suspected disease. During the difficult diagnosis process, multiple auxiliary examination, biopsy and lung lobe puncture were performed to exclude occult malignancy.

Currently, there is no guidance or consensus on the diagnosis of ectopic thyroid with bilateral multiple pulmonary nodules in clinical practice (11). Ectopic thyroid can be positioned in different locations based on thyroid development due to early developmental differentiation. Depending on its location, size and state of malignancy, ectopic thyroid can cause different symptoms. Given that multiple small nodules in the lungs are more likely to be metastasis from another malignancy, defining the nodules by a surgical or traumatic technique is currently considered the most necessary approach, aiding the subsequent treatment (11). It should not be ignored that the treatment strategy and prognosis could vary according to the diagnosis by the histopathological findings of the nodules; thus, the nature of the nodule is a key issue. To clarify the nature of the pulmonary nodule of our patient, a percutaneous lung biopsy was performed to obtain pathological tissue. Surprisingly, pathology IHC confirmed that the nodule was completely consistent with normal thyroid tissue. Ectopic thyroid is one of the least common thyroid congenital abnormalities and is frequently found in the midline, from the tongue to the diaphragm. Although the cause of this abnormality is not very clear, it has been determined that mutations in genetic factors and regulatory genes expressed during thyroid development are linked to ectopic thyroid (12). In the past few decades, only a few sporadic cases of ectopic thyroid in the lung have been reported $(3,13-16)$. The case we reported has a distinct difference compared with other cases, as multiple nodules were accidentally and simultaneously found in bilateral pulmonary lobes without any notable medical history. Of note, it is extremely rare, but not impossible, that benign-like intrapulmonary nodules are actually metastasized from occult thyroid carcinoma. Furthermore, although rare, the development of malignant diseases in ectopic thyroid tissue has also been recognized (17). Some other diseases, such as thymoma, neuroma and germ cell tumours, must also be differentiated from ectopic intrapulmonary thyroid (11). Therefore, the diagnosis of our patient should be made more cautiously, with exclusion of any other kinds of tumours from thyroid or other organs.

Prior to treatment of ectopic thyroid, there are many factors that should be considered when deciding whether to perform surgical and medical treatment. These factors include age, thyroid hormone level, symptoms due to nodules and their severity, and malignancy. For ectopic thyroid gland with symptoms or malignancy history, surgery or radioactive iodine ablation is a common treatment (11). However, it must be considered that the only effective thyroid tissue may be lost when considering the treatment of a prominent ectopic thyroid, especially in the lingual ectopic thyroid gland, which is the only effective thyroid tissue in most patients (8). Thus, treatment decisions should be made very cautiously to avoid delaying so as not to miss the best treatment opportunity and to avoid over-treatment. More importantly, the patient's own wishes must be respected. Recently, multifocal ectopic thyroid tissues in the breast of a 53-year-old woman was reported in Korea (18). Despite the lack of standard therapy due to the condition's rarity, the patient did not show symptoms related to thyroid hormones, and no further surgery was performed with the consent of the patient and her family. Although ectopic thyroid tissue is benign, it has the potential to progress and even undergo tissue transformation. Therefore, regular follow-up is very important.

After thorough discussion and consideration, our patient decided not to receive treatment for the time being, choosing to "watch and wait" and to have regular follow-up examinations in the clinic. During the follow-up period, examinations were performed routinely, including thyroid palpation and function tests, neck B-ultrasound and pulmonary CT. The patient developed no malignant 
symptoms, and the sizes of the intrapulmonary nodules remained the same as at the beginning. Based on these findings, the patient's intrapulmonary nodules are currently considered to be benign ectopic pulmonary thyroid tissue.

Unfortunately, due to the limited initial understanding of the disease, we have not further improved the detection of mutations in genetic factors and regulatory genes linked to ectopic thyroid, such as PAX8 (12). Combined with the existing examination and pathological results, the patient's lung nodules were diagnosed as ectopic thyroid. We will continue to follow-up our patients and track related reports of similar multiple ectopic thyroids. Similar cases will be discussed further to develop better guidance for diagnosis and treatment protocols.

In conclusion, we reported an exceedingly rare case of multiple bilateral pulmonary nodules with normal thyroid function and without history of malignant disease. Different from the other reported cases of ectopic thyroid with active treatment, our patient received no surgery and accepted our "watch and wait" strategy by regularly submitting to followup examinations to avoid over-treatment. Multiple ectopic thyroid nodules may be easily misdiagnosed in the clinic. We believe this is a debate that may provoke the accurate diagnosis and management of relevant cases.

\section{Acknowledgments}

Funding: None.

\section{Footnote}

Conflicts of Interest: All authors have completed the ICMJE uniform disclosure form (available at http://dx.doi. org/10.21037/gs-19-499). The authors have no conflicts of interest to declare.

Ethical Statement: The authors are accountable for all aspects of the work in ensuring that questions related to the accuracy or integrity of any part of the work are appropriately investigated and resolved. Written informed consent was obtained from the patient for publication of this manuscript and any accompanying images.

Open Access Statement: This is an Open Access article distributed in accordance with the Creative Commons Attribution-NonCommercial-NoDerivs 4.0 International License (CC BY-NC-ND 4.0), which permits the noncommercial replication and distribution of the article with the strict proviso that no changes or edits are made and the original work is properly cited (including links to both the formal publication through the relevant DOI and the license). See: https://creativecommons.org/licenses/by-nc-nd/4.0/.

\section{References}

1. Noyek AM, Friedberg J. Thyroglossal duct and ectopic thyroid disorders. Otolaryngol Clin North Am 1981;14:187-201.

2. Noussios G, Anagnostis P, Goulis DG, et al. Ectopic thyroid tissue: anatomical, clinical, and surgical implications of a rare entity. Eur J Endocrinol 2011;165:375-82.

3. Cheng H, Yang L, Xiong J, et al. Multiple thyroid nodules in the lung: metastasis or ectopia? Diagn Pathol 2015;10:61.

4. Li Y, Li S, Wang M, et al. Ectopic thyroid in the gallbladder accompanied with gallbladder adenoma: A case report. Medicine (Baltimore) 2019;98:e18293.

5. Rajabi P, Eftekhari SM, Rouhani E, et al. Ectopic Thyroid in Stomach; a Case Report. Iran J Pathol 2018;13:103-5.

6. Tada A, Tanaka T, Takamoto A, et al. Ectopic thyroid tissue in the adrenal gland: CT and MRI findings. Diagn Interv Imaging 2016;97:373-5.

7. Chen M, Hu J, Cai X. Ectopic Thyroid Gland Tissue in the Liver. Clin Gastroenterol Hepatol 2019;S15423565(19)31093-6.

8. Baik SH, Choi JH, Lee HM. Dual ectopic thyroid. Eur Arch Otorhinolaryngol 2002;259:105-7.

9. Chawla M, Kumar R, Malhotra A. Dual ectopic thyroid: case series and review of the literature. Clin Nucl Med 2007;32:1-5.

10. Gagnier JJ, Kienle G, Altman DG, et al. The CARE Guidelines: Consensus-based Clinical Case Reporting Guideline Development. Glob Adv Health Med 2013;2:38-43.

11. $\mathrm{Xu} \mathrm{G}, \mathrm{Fu} \mathrm{X}$. One-stage video-assisted thoracic surgery for bilateral multiple pulmonary nodules. J Thorac Dis 2019;11:535-41.

12. Ibrahim NA, Fadeyibi IO. Ectopic thyroid: etiology, pathology and management. Hormones (Athens) 2011;10:261-9.

13. Ko HH, Cho SW, Lee HS, et al. Ectopic intrapulmonary thyroid: a case report. Korean J Thorac Cardiovasc Surg 2013;46:237-9.

14. Simon M, Baczako K. Thyroid inclusion in the lung. Metastasis of an occult papillary carcinoma or ectopia? 
Pathol Res Pract 1989;184:263-7.

15. Zhang $W$, Zhang $\mathrm{H}$, Hou Q, et al. Ectopic thyroid microfollicular adenoma in the lung: A case report. Medicine (Baltimore) 2019;98:e16832.

16. Ryu HS, Chung YJ, Chong S, et al. Ectopic intrapulmonary thyroid tissue mimicking metastatic tissue. Thyroid 2012;22:755-9.

Cite this article as: Cheng L, Jiang X, Jiang Y. Ectopic thyroid as multiple nodules in bilateral lung lobes: a case report. Gland Surg 2020;9(3):806-811. doi: 10.21037/gs-19-499
17. Klubo-Gwiezdzinska J, Manes RP, Chia SH, et al. Clinical review: Ectopic cervical thyroid carcinoma--review of the literature with illustrative case series. J Clin Endocrinol Metab 2011;96:2684-91.

18. Moon A, Kim HS, Chang K, et al. Multifocal ectopic thyroid tissues including breast: A case report. Mol Clin Oncol 2020;12:117-9. 